\title{
Effects of the SLCO1B1 A388G single nucleotide polymorphism on the development, clinical parameters and survival of multiple myeloma cases in a Polish population
}

Katarzyna Michalska ( $\sim$ katarzyna.niebudek@umed.lodz.pl )

Medical University of Lodz

Ewa Balcerczak

Medical University of Lodz

Jacek Pietrzak

Medical University of Lodz

Marta Żebrowska-Nawrocka

Medical University of Lodz

\section{Research Article}

Keywords: multiple myeloma, OATP1B1, SNP, polymorphism

Posted Date: May 10th, 2021

DOI: https://doi.org/10.21203/rs.3.rs-470671/v1

License: (c) (i) This work is licensed under a Creative Commons Attribution 4.0 International License. Read Full License 


\section{Abstract}

Multiple myeloma (MM) is a malignant disease of plasma cells with complex etiopathogenesis, causing significant morbidity due to multi-organ damage. Changes in the genes encoding transport proteins, resulting in changes in their function, affect the cell defense potential and response to the chemotherapy applied. The aim of this study was to determine the A388G single nucleotide polymorphism (SNP) in the SLCO1B1 gene in Polish multiple myeloma patients. The material for the study included DNA isolated from nucleus cells of peripheral blood in patients diagnosed with multiple myeloma. Clinicopathological characteristics, treatment processes, laboratory findings, and treatment outcomes were summarized and statistically analyzed. The studied polymorphism does not seem to affect the increased risk of development or treatment outcomes of multiple myeloma. Our research primarily focuses on investigating the association of transporter polymorphisms with multiple myeloma and providing theoretical evidence. Further studies are needed to better understand molecular mechanisms underlying an altered function of organic anion transporting polypeptides (OATPs) in MM.

\section{Introduction}

Multiple myeloma (MM) is a genetically complex plasma cell neoplasm that evolves from pre-malignant stages following genomic evolution which leads to the proliferation of malignant plasma cells ${ }^{1}$. MM is characterized by the clonal B-cell proliferation in bone marrow typically associated with overproduction of monoclonal proteins that accumulate in serum and urine $^{2}$. The clonal expansion of monotypic plasma cells in the bone marrow is often associated with excessive production/secretion of monoclonal protein into the blood ${ }^{3}$. The development of multiple myeloma causes a variety of clinical symptoms, including anemia, hypercalcemia, immune paresis, and organ damage such as kidney and bone disorders ${ }^{2,3}$. Multiple myeloma is one of the most common hematological malignancies in adults worldwide and accounts for $1.8 \%$ of all cancer cases and approximately $10 \%$ of hematologic malignancies ${ }^{3,4}$. In Europe there are more than 48,000 new cases and around 31,000 deaths each year ${ }^{5,6}$. MM is frequently observed in patients of advanced age, approximately $66-70$ years, with a slight male prevalence ${ }^{7}$. Multistep genetic alterations lead to the progression from MGUS - monoclonal gammopathy of undetermined significance to multiple myeloma in some persons. Cytogenetic abnormalities are detected in $90 \%$ of the plasma cells in patients with multiple myeloma ${ }^{8}$.

Over the past two decades, strategies for MM therapy have rapidly evolved and led to improved outcomes including prolonged survival, mainly due to availability and application of new drugs and their combinations with limited toxicity, such as: proteasome inhibitors, immunomodulators, and monoclonal antibodies ${ }^{2,3,9}$. Nevertheless, the disease is considered incurable and displays significant heterogeneity in clinical presentation, course and survival ${ }^{10}$. Epidemiological data encourages the continuous search for new treatments and therapeutic strategies to achieve prolonged survival with a good quality of life and perhaps reach the so far elusive cure of the disease ${ }^{2}$. Moreover, predicting the treatment response of individual patients at the time of diagnosis remains difficult ${ }^{11}$. While the complexity and heterogeneity of the disease continue to make personalized medicine a challenge for myeloma patients, the genetic background research will undoubtedly contribute to more precision medicine in myeloma in the near future. Importantly, in-depth assessment of the genetic background of MM to an improved understanding more completely characterizes the disease, thus the identification of new targets and development of better therapies for myeloma patients are needed ${ }^{1,10}$

To function properly, the human body must constantly transport various substances, both xenobiotics and endogenous compounds, through biological barriers. There are many groups of membrane transporters divided into families due to the homology of structure and the specifics of transported substrates. The main families are as follows: the ABC - ATP-binding cassette family, OATP - organic anion transporting polypeptide family, PepT - peptide transporter family, OAT - organic anion transporter family, and OCT- organic cation transporter family ${ }^{12}$. In humans, the solute carrier (SLC) family of membrane transport proteins consists of approximately 300 individual proteins and is organized into 43 families. The SLC families encode proteins for: passive transporters, ion-coupled transporters and exchangers mediating uptake of substrates into cells ${ }^{12,13}$. The superfamily of organic anion-transporting polypeptides (OATPs, gene symbol SLCO) includes important transporters handling a variety of endogenous and xenobiotic substrates including: organic dyes, bile acids, prostaglandins, cyclic nucleotides, steroid 
hormones and their conjugates, thyroid hormones and environmental toxins ${ }^{14-16}$. OATPs as the main influx drug transporters significantly contribute to the absorption, distribution, and elimination (ADME) of pharmaceutical agents and the involvement of drug-drug interaction (DDI) ${ }^{12,17-19}$. The substrates transported by OATPs are the following: antibiotics, antidiabetic drugs, antiinflammatory drugs, antifungals, antivirals, antihistamines, antihypertensives, fibrates, statins, cardiac glycosides, immunosuppressants, and anticancer drugs, e.g. atorvastatin, cerivastatin, methotrexate, paclitaxel, rapamycin, flavopiridol, SN38, gimatecan, doxorubicin, and docetaxel 12,14,18,20,21. OATPs are expressed in various tissues and organs, such as liver, intestine, blood-brain barrier, kidney, placenta and other organs ${ }^{12,22,23}$. It is now well recognized that certain OATPs are differentially regulated in normal and cancer tissues ${ }^{23}$. There is evidence that the expression of some OATPs may be up- or downregulated in several types of cancers, suggesting their potential pathogenic roles during the development and progression of cancer ${ }^{14,24}$. OATPs expression levels are altered in many different types of cancer and in some have been correlated with cancer stage and outcomes. OATPs are capable of transporting many compounds that affect the growth and survival of cancer cells, including hormones, hormone precursors, and anti-cancer drugs ${ }^{25,26}$. The expression of OATPs transporters in neoplasms may influence the intracellular concentration of drugs, thus influencing their effectiveness. In addition, the expression levels of these influx transporters, known to cooperate with efflux transporters and drug metabolizing enzymes, respectively, may play a key role in chemoresistance mechanisms ${ }^{26}$.

A number of naturally occurring single nucleotide polymorphisms (SNPS) in the genes encoding OATPs have been reported and extensively investigated for their impact on the expression and function of OATP transporters. Several studies have shown that SNPs from OATP are associated with effects on the presence and function of proteins, and some SNPs have been associated with altered chemotherapy drug distribution and consequently increased side effects ${ }^{25}$. In particular, polymorphic variants of genes encoding OATP1A2, OATP1B1, and OATP1B3 have been reported to be clinically relevant ${ }^{14}$. The SLCO1B1 gene composed of 15 exons and 14 introns is located on the short arm of chromosome 12 (gene locus 12p12) and encodes a 691 amino acids protein with 12 transmembrane helices ${ }^{27,28}$. The SLCO1B1 gene spans 15 exons and 190 common variants with minor allele frequency, greater than $5 \%{ }^{28,29}$. Although many SNPs have been identified in SLCO1B1, only several are known to have functional effects and clinical significance, e.g. SLC01B1 rs2306283 (A388G, N130D) or rs4149056 (T521C, V174A) 21,28,30. The A388G and T521T C form four main haplotypes: *1A (388A/521T) - wild-type, *1B (388G/521T), *5 (388A/521C) and $* 15(388 \mathrm{G} / 521 \mathrm{C}){ }^{15,20}$. The clinical importance of $S L C O 1 B 1$, mainly $* 5$ or $* 15$, for statin-induced myopathy is well demonstrated ${ }^{31}$. The A388G (rs2306283) SNP is associated with the altered transport function resulted in changes in the structure of the transmembrane-spanning domains ${ }^{30}$. The $\mathrm{G}$ allele at rs 2306283 (referred to as the *1B variant) causes a substitution that may increase the OATP1B1 function, however the functional consequences of this variant remain controversial 32. Some studies demonstrated that the A388G has an unaltered transport function in in vitro studies, others have shown that the A388G variant is significantly associated with the increased SLCO1B1 expression, suggesting increased functional activity. Absolute protein quantification showed that OATP1B1 protein levels were significantly higher in the c.388 GG genotype vs. the c.388 AA genotype, confirming the increased transport function of N130D-OATP1B1 in vivo ${ }^{15,33}$. Differentially regulated OATPs may have pathogenic roles during cancer development and progression and potentially serve as therapeutic targets in cancer. The A388G in SLCO1B1, known to be associated with the risk of colorectal cancer, was previously investigated regarding its effect on overall survival and time to recurrence in Italian colorectal cancer (CRC) patients followed up after surgery. In the analysis, the risk of death significantly increased by the rare allele for A388G ${ }^{34}$.

Thus, the aim of our work was to determine SLCO1B1 rs2306283 gene polymorphism in multiple myeloma patients. The research may allow us to better understand the molecular mechanisms underlying the altered expression of OATPs, cancer development, anticancer drug transport and therapy efficiency to find out how these transporters can be used as potential molecular markers of the diagnostic, prognostic, and predictive nature or in cancer treatment involving individual response to drugs. To the best of our knowledge, the role of this polymorphism in multiple myeloma has not been studied in the Polish population so far.

\section{Materials And Methods}


The investigated group contained 157 blood samples were collected from patients with multiple myeloma diagnosed at the Department of Hematology, Medical University of Lodz, Poland. Patients with MM were diagnosed according to the International Myeloma Working Group Classification included in the study. Due to the lack of availability of complete clinical-pathological data for all patients, subsequent statistical analyzes were performed with less numerous groups.

The control group consisted of 141 blood samples obtained from healthy individuals from the local blood bank, who geographically and ethnically matched the group of patients with MM. The investigation was carried out in accordance with the principles of the Declaration of Helsinki and was approved by the Ethical Committee of the Medical University of Lodz No: RNN/93/20/KE, RNN/88/16/KE; RNN/285/13/KE. All patients provided a written informed consent before their inclusion in the study.

\section{DNA isolation:}

DNA was isolated from peripheral blood according to the "Blood Mini" protocol (A\&A Biotechnology, Poland). DNA samples, until further analysis, were stored at $-20^{\circ} \mathrm{C}$. DNA quantity and quality/purity were determined photometrically at $260 \mathrm{~nm}$ and $280 \mathrm{~nm}$ using the Nanophotometer (IMPLEN, Germany) according to the manufacturer's instructions.

\section{Genotyping of A388G:}

To determine the A388G (rs2306283) polymorphism in the SLC01B1 gene, the PCR-restriction fragment length polymorphism method (PCR-RFLP) was used. For analyzing the SLCO1B1 variants, the forward primer 5'-CATGCTGGGAAATTGACAGAAAGT-3' and the reverse primer 5'-GAAAACGCGTAGTTTAAACCTGT -3' were used. The PCR reaction was performed in a total reaction

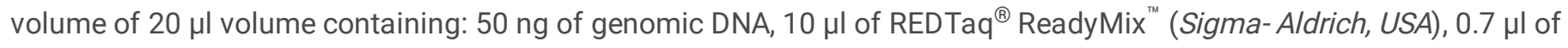
$10 \mu \mathrm{mol}$ of forward and reverse primers and distilled water up to a final volume. The PCR parameters were as follows: an initial denaturation at $94^{\circ} \mathrm{C}$ for $5 \mathrm{~min}$, followed by 35 cycles of $94^{\circ} \mathrm{C}$ for $30 \mathrm{~s}, 58^{\circ} \mathrm{C}$ for $30 \mathrm{~s}$ (annealing), $72^{\circ} \mathrm{C}$ for $30 \mathrm{~s}$ (extension), and an extra extension step at $72^{\circ} \mathrm{C}$ for $5 \mathrm{~min}$. The negative control (without DNA template) was included in every experiment. The PCR product for the A388G SNP of the SLC01B1 gene was $462 \mathrm{bp}$ in size. In the next step, PCR products were digested with the Taq/ restriction enzyme (EURX Sp. z o. o., Poland) at $65^{\circ} \mathrm{C}$ for $16 \mathrm{~h}$. The digested PCR products were separated by electrophoresis using a $3 \%$ agarose gel stained with ethidium bromide and visualized by an UV transilluminator. Electrophoretic analysis of genotypes was performed. The bands patterns presentation was: AA $194+268$, AG $23+171+194+268$, GG $23+171+268$. All samples from the study and control group were successfully analyzed. An exemplary image of the electrophoretic separation is presented in Figure 1.

\section{Statistical analysis:}

The statistical analyses were performed using STATISTICA 13 statistical software (StatSoft Inc. 2018). Differences in genotype and allele frequencies of $\mathrm{A} 388 \mathrm{G}$ among multiple myeloma patients and the control group were determined using the chi-square test. To determine the significance of differences $A 388 \mathrm{G}$ polymorphism and clinical-pathological features of the MM patients, the chi-square test was used. The Kaplan-Meier analysis was carried out to estimate overall survival time (OS). Overall survival was defined as the interval from the date of diagnosis to the date of death or the last clinical appointment. The effects of A388G polymorphism on survival were examined using the chi-square test for genotypes and log-rank test for alleles using proportional hazards model. In all conducted tests, a $p$ value of $<0.05$ was assumed as significant.

\section{Results}

First, the frequencies of alleles and genotypes of the studied A388G SNP (rs2306283) between the investigated and control groups were compared. There were no statistically significant differences in the frequencies of the genotype $(p=0.8211)$ and alleles: $A(p=0.5442)$ and $G(p=0.802)$ between groups. However, the $G G$ genotype occurred slightly more often in the group of MM patients than in the control group ( $28.7 \% ; 25.5 \%$, respectively). The GG genotype was associated with a 1.15 -fold higher incidence of this disease compared to the AG and AA genotypes. The details are presented in Table 1. The A388G variant has 
been seen but significantly less so in European-Americans (30-45\%) ${ }^{15,30,35}$. In our results, the SLCO1B1 388G allele was presented with a lower frequency in the Polish population, similarly to other Caucasians ${ }^{28}$.

Table 1 Frequency of genotype and allele distributions of the A388G SNP between the investigated and control group.

\begin{tabular}{|c|c|c|c|c|}
\hline SLCO1B1 & Multiple myeloma $\mathbf{N}=157$ [\%] & Healthy individuals $\mathrm{N}=141[\%]$ & $\mathrm{p}$ & OR [95\%] \\
\hline \multicolumn{5}{|l|}{ SNP A388G } \\
\hline AA & 79 [50.3] & $73[51.8]$ & \multirow[t]{3}{*}{0.8211} & 1 \\
\hline AG & $33[21.0]$ & $32[22.7]$ & & $0.95[0.53-1.70]$ \\
\hline GG & $45[28.7]$ & $36[25.5]$ & & $1.15[0.67-1.98]$ \\
\hline A present & $191[60.8]$ & $178[63.1]$ & \multirow[t]{2}{*}{0.5442} & \multirow[t]{2}{*}{-} \\
\hline A absent & $123[39.2]$ & $104[36.9]$ & & \\
\hline G present & $123[39.2]$ & $104[36.9]$ & \multirow[t]{2}{*}{0.802} & \multirow[t]{2}{*}{ - } \\
\hline G absent & $191[60.8]$ & $178[63.1]$ & & \\
\hline
\end{tabular}

The further performed statistical analyzes depended on the availability of clinical-pathological data. The group of patients with multiple myeloma was divided according to gender. Among the group of $97 \mathrm{MM}$ patients, 47 were men (48.5\%) and 50 were women (51.5\%). Then the association between gender and the prevalence of individual genotypes and alleles for A388G polymorphism in the SLCO1B1 gene was analyzed. No statistical significance was found for genotypes and also for the presence of the A allele or the $\mathrm{G}$ allele $(\mathrm{p}=0.9147 ; \mathrm{p}=0.6738$ and $\mathrm{p}=0.7813)$, respectively.

The next comparable parameter was age. The median age of MM patients $(N=79)$ at diagnosis was 63 years (range, 40 to 87 years). The patients were divided into two subgroups: the first subgroup of patients aged $\leq 63$ years and the second subgroup aged over 63 years. The genotype AA tended to occur more frequently in the subgroup of patients aged $\leq 63$ years $(p=0.0742)$. The allele analysis showed that the occurrence of at least one A allele was statistically significantly more frequent $(p=0.0357)$ in the subgroup of patients under 63 years of age (79\%) than in the subgroup over 63 years (58\%).

Many laboratory parameters allow clinicians to monitor the disease progress, assessing the effectiveness of treatment and prognosis in multiple myeloma. In the course of $\mathrm{MM}$, characteristic changes in the results of laboratory tests are observed. The most common abnormality in multiple myeloma is anemia, i.e. a reduced number of red blood cells and hemoglobin levels. Likewise, the elevated creatinine levels are observed which indicate a worse functioning of the kidneys as a result of damage by monoclonal proteins. At the next stage of the analysis, the MM group was divided into subgroups according to hemoglobin $(\mathrm{N}=61)$ and creatinine $(\mathrm{N}=59)$ levels. In the case of the hemoglobin level $(\mathrm{Hb})$, the first subgroup of patients had it lower than or equal to $9.2 \mathrm{~g} / \mathrm{dL}$, the second subgroup had the $\mathrm{Hb}$ level over $9.2 \mathrm{~g} / \mathrm{dL}$; and as regards the creatinine level, the first subgroup had it lower than or equal to $2 \mathrm{mg} / \mathrm{dL}$, the second subgroup had this level over $2 \mathrm{mg} / \mathrm{dL}$. When we compared the distribution of the A388G SNP according to the hemoglobin level in the subgroups of MM patients, no significant differences for investigated genotypes and alleles were observed $(p=0.2020)$. However, the $A$ allele had the tendency to be more frequent in the subgroup of patients with the hemoglobin level lower or equal to $9.2 \mathrm{~g} / \mathrm{dL}(\mathrm{p}=0.0771)$. In the case of the creatinine level analysis, no statistical significance was demonstrated $(p=0.7133)$.

Further analysis according to the stage of advancement in line with the Durie-Salmon classification was performed ( $N=52)$. In this part of the analysis also no significant differences were observed in the genotype frequencies $(p=0.2075)$. This is worth noting that at least one A allele was more frequent in the subgroup of patients who were classified as stage III ( $80 \%)$ than in stage I $(60 \%)$ or stage II (43\%) according to the Durie-Salmon classification $(p=0.0974)$.

The data on the type of scheme of chemotherapy used during the treatment was available for 77 multiple myeloma patients. The Melphalan-Prednisone - MP scheme was applied in 28 (36 \%) cases, Vincristine-Adriamycin-Dexamethasone - VAD scheme was used in 41 (53 \%) cases; in the remaining cases, different treatment regimens were used. No statistical significance was 
demonstrated in the analysis concerning the association between the A388G SNP and the treatment scheme. The distributions of genotype and allele frequencies of the analyzed clinical-pathological features are summarized in Table 2.

Table 2 The frequency of the studied A388G SNP in patients with multiple myeloma according to the clinicalpathological features.

\begin{tabular}{|c|c|c|c|c|c|c|c|c|c|c|c|c|}
\hline & \multirow[t]{2}{*}{$\mathbf{N}$} & \multicolumn{10}{|c|}{ Prevalence of the investigated A388G SNP in multiple myeloma patients } \\
\hline & & & $\begin{array}{l}\text { AA } \\
\text { [\%] }\end{array}$ & $\begin{array}{l}\text { AG } \\
{[\%]}\end{array}$ & GG [\%] & $\mathrm{p}$ & $\begin{array}{c}\mathbf{A} \\
\text { present } \\
{[\%]}\end{array}$ & \begin{tabular}{|c}
$\mathbf{A}$ \\
absent \\
{$[\%]$}
\end{tabular} & $\mathrm{p}$ & $\begin{array}{c}\mathrm{G} \\
\text { present } \\
{[\%]}\end{array}$ & $\begin{array}{c}\text { G } \\
\text { absent } \\
{[\%]}\end{array}$ & p \\
\hline \multirow[t]{2}{*}{ Gender } & Female & 50 & $28[56]$ & $8[16]$ & $14[28]$ & \multirow[t]{2}{*}{0.9147} & $36[72]$ & $14[28]$ & \multirow[t]{2}{*}{0.6738} & $22[44]$ & $28[56]$ & \multirow[t]{2}{*}{0.7813} \\
\hline & Male & 47 & 25 [53] & $7[15]$ & $15[32]$ & & $32[68]$ & $15[32]$ & & $22[47]$ & 25 [53] & \\
\hline \multirow[t]{2}{*}{ Age } & $\begin{array}{l}<=63 \\
\text { years }\end{array}$ & 39 & 26 [67] & 5 [12] & $8[21]$ & \multirow[t]{2}{*}{0.0742} & 31 [79] & $8[21]$ & \multirow[t]{2}{*}{0.0357} & 13 [33] & 26 [67] & \multirow[t]{2}{*}{0.0311} \\
\hline & $\begin{array}{l}>63 \\
\text { years }\end{array}$ & 40 & 17 [43] & $6[14]$ & 17 [43] & & $23[58]$ & 17 [42] & & $23[58]$ & $17[42]$ & \\
\hline \multirow[t]{2}{*}{ Hemoglobin } & $\begin{array}{c}<=9.2 \\
\mathrm{~g} / \mathrm{dL}\end{array}$ & 31 & 20 [65] & $5[16]$ & $6[19]$ & \multirow[t]{2}{*}{0.2020} & 25 [80] & $6[20]$ & \multirow[t]{2}{*}{0.0771} & $11[34]$ & 20 [65] & \multirow[t]{2}{*}{0.2517} \\
\hline & $\begin{array}{l}>9.2 \\
\mathrm{~g} / \mathrm{dL}\end{array}$ & 30 & 15 [50] & $3[10]$ & $12[40]$ & & $18[60]$ & $12[40]$ & & $15[50]$ & $15[50]$ & \\
\hline \multirow{3}{*}{$\begin{array}{c}\text { Stage of } \\
\text { advancement } \\
\text { according to } \\
\text { Durie-Salmon }\end{array}$} & I & 5 & $3[60]$ & $0[0]$ & $2[40]$ & \multirow[t]{3}{*}{0.2075} & $3[60]$ & $2[40]$ & \multirow[t]{3}{*}{0.0974} & $2[40]$ & $3[60]$ & \multirow[t]{3}{*}{0.6203} \\
\hline & II & 7 & $3[43]$ & $0[0]$ & $4[57]$ & & 3 [43] & 4 [57] & & 4 [57] & $3[43]$ & \\
\hline & III & 40 & 25 [63] & 7 [17] & $8[20]$ & & $32[80]$ & $8[20]$ & & 15 [37] & 25 [63] & \\
\hline \multirow{2}{*}{$\begin{array}{c}\text { Creatinine } \\
>=2 \mathrm{mg} / \mathrm{dL}\end{array}$} & No & 44 & 24 [55] & 6 [13] & 14 [32] & \multirow[t]{2}{*}{0.7689} & 30 [68] & 14 [32] & \multirow[t]{2}{*}{0.9136} & 20 [45] & 24 [55] & \multirow[t]{2}{*}{0.7133} \\
\hline & Yes & 15 & 9 [60] & $1[7]$ & 5 [33] & & $10[67]$ & 5 [33] & & $6[40]$ & $9[60]$ & \\
\hline \multirow{3}{*}{$\begin{array}{c}\text { Type of } \\
\text { chemotherapy }\end{array}$} & MP* & 28 & 12 [43] & $6[21]$ & $10[36]$ & \multirow[t]{3}{*}{0.6707} & 18 [64] & 10 [36] & \multirow[t]{3}{*}{0.3574} & 16 [57] & 12 [43] & \multirow[t]{3}{*}{0.4569} \\
\hline & VAD** & 41 & 23 [56] & $8[20]$ & $10[24]$ & & 31 [78] & 10 [22] & & $18[44]$ & 23 [56] & \\
\hline & Other**** & 8 & $5[63]$ & 1 [17] & $2[25]$ & & $7[88]$ & $1[12]$ & & $3[37]$ & $5[63]$ & \\
\hline
\end{tabular}

\author{
*MP- Melphalan-Prednisone \\ **VAD - Vincristine-Adriamycin-Dexamethasone \\ *** Other: Melphalan /or Bortezomib/or Bortezomib + VAD
}

There are different types of myeloma, classified according to the type of immunoglobulins (Ig) produced by the myeloma cells. The most common type of myeloma is IgG with a gamma immunoglobulin heavy chain. About $60 \%$ of people with multiple myeloma have IgG, while about $30 \%$ have the rarer types: IgA, IgD, IgE and IgM or an antibody fragment: kappa or lambda light chains. The type of myeloma diagnosis does not usually influence treatment, but it can affect the course of the disease in an individual patient. For 78 trials, clinical data was given about the type of immunoglobulins secreted by myeloma cells. The group of patients with MM was divided into three subgroups according to the type of the produced immunoglobulins. The produced immunoglobulin subtype was IgG for 46 patients (59\%), IgA for 17 patients (22\%), and light chains for 15 patients (19\%). Also, in this case, no statistical association was found between the different genotypes and alleles of the A388G SNP of the SLCO1B1 gene and the type of produced immunoglobulins $(p=0.6939)$. Details in Table 3.

Table 3 Prevalence of genotypes and alleles of the A388G SNP in the SLCO1B1 gene in patients with multiple myeloma according to the type of immunoglobulins secreted by myeloma cells.

\begin{tabular}{|c|c|c|c|c|}
\hline \multirow{3}{*}{ SLCO1B1 } & \multicolumn{3}{|c|}{ Multiple myeloma patients N=78 } & \multirow{2}{*}{$\mathbf{p}$} \\
\cline { 2 - 4 } & \multicolumn{2}{|c|}{ Immunoglobulin subtype } & \\
\cline { 2 - 4 } & IgG [\%] & IgA [\%] & Light chains [\%] & \\
\hline AA & $27[34.6]$ & $7[9.0]$ & $8[10.2]$ & \multirow{2}{*}{0.6939} \\
\hline AG & $14[17.9]$ & $6[7.7]$ & $5[6.4]$ & \\
\hline GG & $5[6.4]$ & $4[5.1]$ & $2[2.6]$ & \\
\hline A present & $32[41.1]$ & $11[14.1]$ & $10[12.8]$ & 0.9284 \\
\hline A absent & $14[17.9]$ & $6[7.7]$ & $5[6.4]$ & \\
\hline G present & $19[24.3]$ & $10[12.8]$ & $7[9.0]$ & 0.4642 \\
\hline A absent & $27[34.7]$ & $7[9.0]$ & $8[10.2]$ & \\
\hline
\end{tabular}


As a last part, the dependence of the genotype on polymorphism at position A388G of the SLCO1B1 gene with the probability of overall survival time (OS) was analyzed. The Kaplan-Meier plot shows the probability of survival in the group of patients with multiple myeloma from the diagnosis to last follow-up. There was no statistically significant difference in survival according to genotypes or the presence of at least one $A$ or $G$ allele $(p=0.1192 ; p=0.3122 ; p=0.5587$, respectively). However, the time of survival was shorter in the subgroup of patients with the AA genotype (median: 321 days) compared to the subgroups of patients with the GG genotype (median: 628 days) or the AG genotype (median: 526 days) (Figure 2). This is confirmed by the results of the analysis for A388G SNP alleles, where the time of survival was shorter in the presence of at least one A allele (A allele present: median 379 days; $A$ allele absent: median 526 days) (Figure 3), and it was longer in the presence of at least one $G$ allele (G allele present: median 597 days; $\mathrm{G}$ allele absent: median 321 days) (Figure 4).

\section{Discussion}

OATPs are membrane proteins that mediate the sodium-independent uptake of a wide range of amphipathic endogenous compounds and many xenobiotics, thus ensuring the regulation of delivery of required substrates and thereby cellular homeostasis ${ }^{19,22}$. To function properly, cells must constantly transport various substances, both xenobiotics and endogenous compounds, across biological barriers. Changes in the amount and / or activity of transport proteins have numerous consequences, e.g. they affect the cell defense potential by regulating the amount of harmful substances in the cell, and lead to cell damage, mutation and oncogenesis. On the other hand, the level of protein activity is usually related to the response to the chemotherapy administered ${ }^{36}$.

To date, most studies have emphasized the investigation of OATP expression in solid tumors. Previous reports revealed that the expression of certain OATPs may be altered in different disease conditions, including many different types of cancers. OATPs have been found to be overexpressed in a variety of human solid tumors, including breast, liver, colon, pancreatic, and ovarian cancers. In several cancers, an altered expression of OATP levels has been correlated with cancer stage and clinical outcomes, suggesting potential roles for OATPs in tumor development and progression and their potential role as novel targets for cancer therapy $14,17,23,25,30,37,38$. OATPs are capable of transporting multiple compounds which affect cancer cell growth and survival, including hormones, hormone precursors, and anticancer drugs ${ }^{25}$. Recently, Chen et al. showed that the OATP1B3 expression was significantly reduced in neoplastic tissues compared to that in adjacent non-neoplastic tissues. Moreover, the OATP1B3 lower expression was significantly correlated with the tumor size, relapse, tumor differentiation, and tumor node metastasis (TNM) rate in hepatocellular carcinoma ${ }^{39}$.

The expression, substrate specificity, and activity of OATP transporters in tumors may affect the intracellular concentration of drugs, and, therefore, influence their effectiveness. OATP1B1 mediates hepatic uptake of many drugs and can influence transporter-mediated drug-drug-interactions (DDIs), therefore is responsible for the multiple side effects of multi-drug therapy, often used in cancer treatment ${ }^{40}$. Furthermore, expression levels of these influx transporters may play a crucial role in chemoresistance mechanisms ${ }^{26}$. Patients with OATP polymorphisms have been found to have altered pharmacokinetics due to their impact on absorption, distribution, and excretion of anticancer drugs, thus cancer outcomes ${ }^{15,23,24,41}$.

Availability of results on the role of polymorphisms in these important transporters in cancers is limited, particularly in the case of hematologic malignancies. Some single nucleotide polymorphisms (SNPs) in the genes encoding OATPs have been reported to be clinically relevant and have been extensively investigated for their impact on the expression, reduction function or absent protein ${ }^{17}$. Therefore, the aim of this study was to assess the potential impact of the one of most common functional A388G SNP variant in SLCO1B1 gene on the risk of multiple myeloma development and outcomes.

Frequencies of SLCO1B1 variants vary among geographical regions ${ }^{30}$. In our research, the G allele prevalence was close to the frequencies reported in other Caucasian populations: $39.2 \%$ in the multiple myeloma group and $36.9 \%$ in the control group. The obtained results are consistent with the data published by Nagy et al. where the frequency of the G allele in the A388G SNP of the SLC01B1 gene was $36.2 \%$ in Hungarian populations ${ }^{28}$. 
The study showed that the AA genotype and the A allele were more common in the control population, while the GG genotype and the $\mathrm{G}$ allele were more common in the group of patients with multiple myeloma. However, the obtained results did not show a statistically significant association between the studied polymorphism and the risk of multiple myeloma $(p=0.8211)$. Additionally, no important association with clinical-pathological features was found. Statistical significance was found only for the presence of at least one A allele and age $(p=0.0357)$. The studied polymorphism has not been verified in multiple myeloma or in other hematologic neoplasms so far. Therefore, we are not able to relate our results to other studies. Our results can only be compared with those obtained in studies on solid tumors. Falkowski et al. have shown that the A388G variant genotypes of SLCO1B1 were not associated with colorectal cancer (CRC); similar results were obtained by Özhan et al. in colorectal cancer 24,42. In another study on two common polymorphisms of OATP4A1, no association with CRC predisposition and tumor recurrence was found ${ }^{41}$.

In the presented study, the dependence of the A388G in the SLCO1B1 gene with the probability of overall survival time has been assessed. The OS was longer if the $\mathrm{G}$ allele was present in the genotype, however there was no statistically significant difference in survival according to genotypes or alleles. Our results were comparable with those obtained by Zhang X. et al., in which there was no difference in overall survival between wild-type and carrier groups of SLCO1B1 A388G in breast cancer patients ${ }^{43}$. On the contrary, Teft et al. have found that progression-free survival (PFS) was significantly longer in SLC01B1 388G/G colorectal cancer patients after irinotecan-based chemotherapy ${ }^{44}$. Therefore, these results can confirm that the presence of the $G$ allele influencing the increase in the expression of the protein responsible for the intracellular transport of chemotherapeutic agents leads to more efficient transport and a higher concentration of the drug in the cell, which makes therapy drug more effective.

There is no full consensus on the effects of A388G mutations on the OATP1B1 transport protein. Although there are conflicting results regarding related with SNPs changes in transport activity, in most studies, the G allele of A388G variant in SLCO1B1 was associated with increased OATP1B1 activity and decreased plasma drug concentrations ${ }^{45}$. Some studies revealed that OATP1B1 could enhance the transport of drugs by transporters, and an in vivo experiment reached the same conclusion ${ }^{20}$. The discrepancy in some results may be due to the differences in ethnicity, as SLCO1B1 allele frequencies are known to vary markedly between different populations.

Most of the research has been devoted to the role of transporters, polymorphic variants and haplotypes in the pharmacokinetics of drugs, including chemotherapeutic agents used in the treatment of hematological malignancies ${ }^{46-48}$. The recent study in adult patients with hematologic malignancies receiving high-dose methotrexate suggests that patients with the SLCO1B1 A388G or T521C variants exhibit differential metabolomic profiles that may modulate the risk for methotrexate induced toxicities. Similar findings have been reported in cancer patients treated with irinotecan, the plasma concentration of active metabolite SN38 was higher and the risk of severe neutropenia was increased by T521C, while the A388G variant does not affect transport activity for SN-38 ${ }^{14,48,49}$. Bortezomib is the first-in-class proteasome inhibitor for the treatment of multiple myeloma. Alam et al. in an in vivo study investigated that bortezomib has low potential to cause OATP-mediated clinical drug-drug interactions (DDIs) 40 .

The expression, polymorphisms, substrate spectrum, importance in drug transport, DDIs, multi-drug resistance mechanisms, turn out to be not the only interesting OATP application in medicine ${ }^{50}$. Zhang $\mathrm{H}$. et al. presented a next different view on the usefulness of OATP transporters in cancer. They have shown that by actively transporting OATPs, which are overexpressed in many types of cancer cells, the diagnostic substance could effectively penetrate cell membranes, rather than normal cells. The results may contribute to the development of a promising diagnostic tool for the differentiation of cancer cells in the early stages of diagnosis ${ }^{51}$.

Intensified studies are necessary to obtain more comprehensive profiles of OATPs differentially regulated in cancer cells and further investigate the role of OATP in multiple myeloma. This will allow researchers to better understand molecular mechanisms underlying an altered expression of OATPs in hematologic cancer development, anticancer drug transport and therapy efficiency to determine how these transporters can be used as potential molecular markers. Further analyzes of polymorphic variants in OATP transporters including haplotype analyzes are planned in the near future. 


\section{Conclusion}

Our study has shown that A388G SNP of the SLC01B1 gene does not predispose to an increased individual risk of developing multiple myeloma or influence the overall survival time. We are aware of the limitations of our study, particularly in decreasing the number of cases studied, especially after their classification according to clinical-pathological parameters. The resulting groups were small and may have limited the possibility of detecting the significance of the studied SNP in MM and drawing final conclusions. In the presented research, we have observed some trends that are not statistically significant. Nonetheless, they still allowed us to perform a statistical analysis. Our research primarily focuses on investigating the association of transporter polymorphisms with multiple myeloma and providing theoretical evidence. Further studies are necessary to obtain more comprehensive profiles of OATPs differentially regulated in cancer cells, along with a better understanding of molecular mechanisms underlying the altered function of OATPs in cancer.

\section{Declarations}

\section{Funding:}

Research supported by statutory funds of the Department of Pharmaceutical Biochemistry and Molecular Diagnostics, Medical University of Lodz, 503/3-015-02/503-31-001.

\section{Conflict of interest:}

The authors declare that they have no known competing financial interests or personal relationships that could have appeared to influence the work reported in this paper.

\section{Availability of data and material:}

The datasets generated during and/or analyzed during the current study are available from the corresponding author on reasonable request. Correspondence and requests for data should be addressed to K. M.

\section{Code availability:}

Not applicable.

\section{Authors' contributions:}

All authors contributed to the study conception and design. KM and EB participated in research design and supervision of the project. KM and JP conducted the experiments. MZ-N and JP contributed to data analysis. KM, MZ-N, EB wrote the manuscript with substantial intellectual contributions from all authors. All authors read and approved the final manuscript.

\section{Ethical approval:}

The investigation was performed in accordance with the Declaration of Helsinki and the Good Laboratory Practice rules and was approved by the Ethical Committee of the Medical University of Lodz No: No: RNN/93/20/KE, RNN/88/16/KE; RNN/285/13/KE.

\section{Consent to participate:}

All patients provided a written informed consent before their inclusion in the study.

\section{Consent to publish:}

Not applicable.

\section{References}


1 Castaneda, O. \& Baz, R. Multiple Myeloma Genomics - A Concise Review. Acta Med Acad 48, 57-67, doi:10.5644/ama2006-124.242 (2019).

2 Legarda, M. A., Cejalvo, M. J. \& de la Rubia, J. Recent Advances in the Treatment of Patients with Multiple Myeloma. Cancers (Basel) 12, doi:10.3390/cancers12123576 (2020).

3 Chen, D., Yang, X., Liu, M., Zhang, Z. \& Xing, E. Roles of miRNA dysregulation in the pathogenesis of multiple myeloma. Cancer Gene Ther, doi:10.1038/s41417-020-00291-4 (2021).

4 Wang, S. et al. IL-17A Increases Multiple Myeloma Cell Viability by Positively Regulating Syk Expression. Trans/ Onco/ 12, 1086-1091, doi:10.1016/j.tranon.2019.04.023 (2019).

5 Ferlay, J. et al. Cancer incidence and mortality patterns in Europe: Estimates for 40 countries and 25 major cancers in 2018. Eur J Cancer 103, 356-387, doi:10.1016/j.ejca.2018.07.005 (2018).

6 Siegel, R. L., Miller, K. D. \& Jemal, A. Cancer statistics, 2020. CA Cancer J Clin 70, 7-30, doi:10.3322/caac.21590 (2020).

7 Riccomi, G., Fornaciari, G. \& Giuffra, V. Multiple myeloma in paleopathology: A critical review. Int J Paleopatho/ 24, 201212, doi:10.1016/j.jpp.2018.12.001 (2019).

8 Michels, T. C. \& Petersen, K. E. Multiple Myeloma: Diagnosis and Treatment. Am Fam Physician 95, 373-383 (2017).

9 Mateos, M. V. et al. Insights on Multiple Myeloma Treatment Strategies. Hemasphere 3, e163, doi:10.1097/hs9.0000000000000163 (2019).

10 Terragna, C. et al. The genetic and genomic background of multiple myeloma patients achieving complete response after induction therapy with bortezomib, thalidomide and dexamethasone (VTD). Oncotarget 7, 9666-9679, doi:10.18632/oncotarget.5718 (2016).

11 Kasamatsu, T. et al. IL17A and IL23R gene polymorphisms affect the clinical features and prognosis of patients with multiple myeloma. Hematol Oncol 36, 196-201, doi:10.1002/hon.2469 (2018).

12 Roth, M., Obaidat, A. \& Hagenbuch, B. OATPs, OATs and OCTs: the organic anion and cation transporters of the SLCO and SLC22A gene superfamilies. Br J Pharmaco/ 165, 1260-1287, doi:10.1111/j.1476-5381.2011.01724.x (2012).

13 Hagenbuch, B. \& Meier, P. J. Organic anion transporting polypeptides of the OATP/ SLC21 family: phylogenetic classification as OATP/ SLCO superfamily, new nomenclature and molecular/functional properties. Pflugers Arch 447, 653-665, doi:10.1007/s00424-003-1168-y (2004).

14 Thakkar, N., Lockhart, A. C. \& Lee, W. Role of Organic Anion-Transporting Polypeptides (OATPs) in Cancer Therapy. Aaps j 17, 535-545, doi:10.1208/s12248-015-9740-x (2015).

15 Lee, H. H. \& Ho, R. H. Interindividual and interethnic variability in drug disposition: polymorphisms in organic anion transporting polypeptide 1B1 (OATP1B1; SLC01B1). Br J Clin Pharmaco/ 83, 1176-1184, doi:10.1111/bcp.13207 (2017).

16 Hagenbuch, B. \& Meier, P. J. The superfamily of organic anion transporting polypeptides. Biochim Biophys Acta 1609, 118, doi:10.1016/s0005-2736(02)00633-8 (2003).

17 Zhou, F., Zhu, L., Wang, K. \& Murray, M. Recent advance in the pharmacogenomics of human Solute Carrier Transporters (SLCs) in drug disposition. Adv Drug Deliv Rev 116, 21-36, doi:10.1016/j.addr.2016.06.004 (2017).

18 Lin, X. et al. Interaction of swine organic anion transporting polypeptide $1 \mathrm{a} 2$ with tetracycline, macrolide and $\beta$-lactam antibiotics. Toxicol Appl Pharmaco/379, 114649, doi:10.1016/j.taap.2019.114649 (2019). 
19 Alam, K. et al. Regulation of Organic Anion Transporting Polypeptides (OATP) 1B1- and OATP1B3-Mediated Transport: An Updated Review in the Context of OATP-Mediated Drug-Drug Interactions. Int J Mol Sci 19, doi:10.3390/ijms19030855 (2018).

20 Gao, C. M. et al. Effect of OATP1B1 genetic polymorphism on the uptake of tamoxifen and its metabolite, endoxifen. Oncol Rep 38, 1124-1132, doi:10.3892/or.2017.5727 (2017).

21 Gregory, B. J., Chen, S. M., Murphy, M. A., Atchley, D. H. \& Kamdem, L. K. Impact of the OATP1B1 c.521T>C single nucleotide polymorphism on the pharmacokinetics of exemestane in healthy post-menopausal female volunteers. $J$ Clin Pharm Ther 42, 547-553, doi:10.1111/jcpt.12569 (2017).

22 Wang, X., Wolkoff, A. W. \& Morris, M. E. Flavonoids as a novel class of human organic anion-transporting polypeptide OATP1B1 (OATP-C) modulators. Drug Metab Dispos 33, 1666-1672, doi:10.1124/dmd.105.005926 (2005).

23 Schulte, R. R. \& Ho, R. H. Organic Anion Transporting Polypeptides: Emerging Roles in Cancer Pharmacology. Mol Pharmaco/ 95, 490-506, doi:10.1124/mol.118.114314 (2019).

24 Falkowski, S. et al. Common variants in glucuronidation enzymes and membrane transporters as potential risk factors for colorectal cancer: a case control study. BMC Cancer 17, 901, doi:10.1186/s12885-017-3728-0 (2017).

25 Obaidat, A., Roth, M. \& Hagenbuch, B. The expression and function of organic anion transporting polypeptides in normal tissues and in cancer. Annu Rev Pharmacol Toxicol 52, 135-151, doi:10.1146/annurev-pharmtox-010510-100556 (2012).

26 Buxhofer-Ausch, V. et al. Tumor-specific expression of organic anion-transporting polypeptides: transporters as novel targets for cancer therapy. J Drug Deliv 2013, 863539, doi:10.1155/2013/863539 (2013).

27 Pu, Z., Zhang, X., Chen, Q., Yuan, X. \& Xie, H. Establishment of an expression platform of OATP1B1 388GG and 521CC genetic polymorphism and the therapeutic effect of tamoxifen in MCF-7 cells. Oncol Rep 33, 2420-2428, doi:10.3892/or.2015.3864 (2015).

28 Nagy, A. et al. Marked differences in frequencies of statin therapy relevant SLCO1B1 variants and haplotypes between Roma and Hungarian populations. BMC Genet 16, 108, doi:10.1186/s12863-015-0262-4 (2015).

29 Romaine, S. P., Bailey, K. M., Hall, A. S. \& Balmforth, A. J. The influence of SLC01B1 (OATP1B1) gene polymorphisms on response to statin therapy. Pharmacogenomics J 10, 1-11, doi:10.1038/tpj.2009.54 (2010).

30 Sissung, T. M. et al. Transporter pharmacogenetics: transporter polymorphisms affect normal physiology, diseases, and pharmacotherapy. Discov Med 13, 19-34 (2012).

31 Aklillu, E. et al. Frequency of the SLC01B1 388A $>$ G and the 521T $>C$ polymorphism in Tanzania genotyped by a new LightCycler®-based method. Eur J Clin Pharmacol 67, 1139-1145, doi:10.1007/s00228-011-1065-9 (2011).

32 Liutkeviciene, R. et al. Evaluation of serum SLC01B1 levels and genetic variants of SLC01B1 rs4149056 and rs2306283 in patients with early and exudative age-related macular degeneration. Gene 676, 139-145, doi:10.1016/j.gene.2018.07.031 (2018).

33 Crowe, A. et al. Characterization of Plasma Membrane Localization and Phosphorylation Status of Organic Anion Transporting Polypeptide (OATP) 1B1 c.521 T>C Nonsynonymous Single-Nucleotide Polymorphism. Pharm Res 36, 101, doi:10.1007/s11095-019-2634-3 (2019).

34 Noci, S. et al. A subset of genetic susceptibility variants for colorectal cancer also has prognostic value.

Pharmacogenomics J 16, 173-179, doi:10.1038/tpj.2015.35 (2016).

35 Pasanen, M. K., Neuvonen, P. J. \& Niemi, M. Global analysis of genetic variation in SLC01B1. Pharmacogenomics 9, 1933, doi:10.2217/14622416.9.1.19 (2008).

Page $11 / 15$ 
36 Basmaci, C. et al. Effects of TNFalpha, NOS3, MDR1 Gene Polymorphisms on Clinical Parameters, Prognosis and Survival of Multiple Myeloma Cases. Asian Pac J Cancer Prev 17, 1009-1014, doi:10.7314/apjcp.2016.17.3.1009 (2016).

37 Hagenbuch, B. \& Stieger, B. The SLCO (former SLC21) superfamily of transporters. Mol Aspects Med 34, 396-412, doi:10.1016/j.mam.2012.10.009 (2013).

38 Green, S. M. et al. Role of OATP transporters in steroid uptake by prostate cancer cells in vivo. Prostate Cancer Prostatic Dis 20, 20-27, doi:10.1038/pcan.2016.42 (2017).

39 Chen, S. et al. Low expression of organic anion-transporting polypeptide 1B3 predicts a poor prognosis in hepatocellular carcinoma. World J Surg Oncol 18, 127, doi:10.1186/s12957-020-01891-y (2020).

40 Alam, K., Farasyn, T., Crowe, A., Ding, K. \& Yue, W. Treatment with proteasome inhibitor bortezomib decreases organic anion transporting polypeptide (OATP) 1B3-mediated transport in a substrate-dependent manner. PLoS One 12, e0186924, doi:10.1371/journal.pone.0186924 (2017).

41 Buxhofer-Ausch, V. et al. Two common polymorphic variants of OATP4A1 as potential risk factors for colorectal cancer. Oncol Lett 20, 252, doi:10.3892/ol.2020.12115 (2020).

42 Ozhan, G., Kara, M., Sari, F. M., Yanar, H. T. \& Alpertunga, B. Influence of the functional polymorphisms in the organic anion transporting polypeptide 1B1 in the susceptibility to colorectal cancer. Genet Test Mol Biomarkers 17, 214-218, doi:10.1089/gtmb.2012.0334 (2013).

43 Zhang, X. et al. Association of CYP2D6*10, OATP1B1 A388G, and OATP1B1 T521C polymorphisms and overall survival of breast cancer patients after tamoxifen therapy. Med Sci Monit 21, 563-569, doi:10.12659/msm.893473 (2015).

44 Teft, W. A. et al. OATP1B1 and tumour OATP1B3 modulate exposure, toxicity, and survival after irinotecan-based chemotherapy. Br J Cancer 112, 857-865, doi:10.1038/bjc.2015.5 (2015).

45 Shitara, Y. et al. Clinical significance of organic anion transporting polypeptides (OATPs) in drug disposition: their roles in hepatic clearance and intestinal absorption. Biopharm Drug Dispos 34, 45-78, doi:10.1002/bdd.1823 (2013).

46 Sai, K. et al. Additive effects of drug transporter genetic polymorphisms on irinotecan pharmacokinetics/pharmacodynamics in Japanese cancer patients. Cancer Chemother Pharmaco/66, 95-105, doi:10.1007/s00280-009-1138-y (2010).

47 Lopez-Lopez, E. et al. Polymorphisms of the SLC01B1 gene predict methotrexate-related toxicity in childhood acute lymphoblastic leukemia. Pediatr Blood Cancer 57, 612-619, doi:10.1002/pbc.23074 (2011).

48 Han, J. Y. et al. Influence of the organic anion-transporting polypeptide 1B1 (OATP1B1) polymorphisms on irinotecanpharmacokinetics and clinical outcome of patients with advanced non-small cell lung cancer. Lung Cancer 59, 69-75, doi:10.1016/j.lungcan.2007.07.019 (2008).

49 Martinez, D. et al. Endogenous Metabolites-Mediated Communication Between OAT1/OAT3 and OATP1B1 May Explain the Association Between SLC01B1 SNPs and Methotrexate Toxicity. Clin Pharmacol Ther 104, 687-698, doi:10.1002/cpt.1008 (2018).

50 Na Nakorn, C. et al. Genetic Variations and Frequencies of the Two Functional Single Nucleotide Polymorphisms of SLC01B1 in the Thai Population. Front Pharmacol 11, 728, doi:10.3389/fphar.2020.00728 (2020).

51 Zhang, H. et al. Dual-channel fluorescence diagnosis of cancer cells/tissues assisted by OATP transporters and cysteine/glutathione. Chem Sci 9, 3209-3214, doi:10.1039/c7sc05407f (2018).

\section{Figures}

Page 12/15 


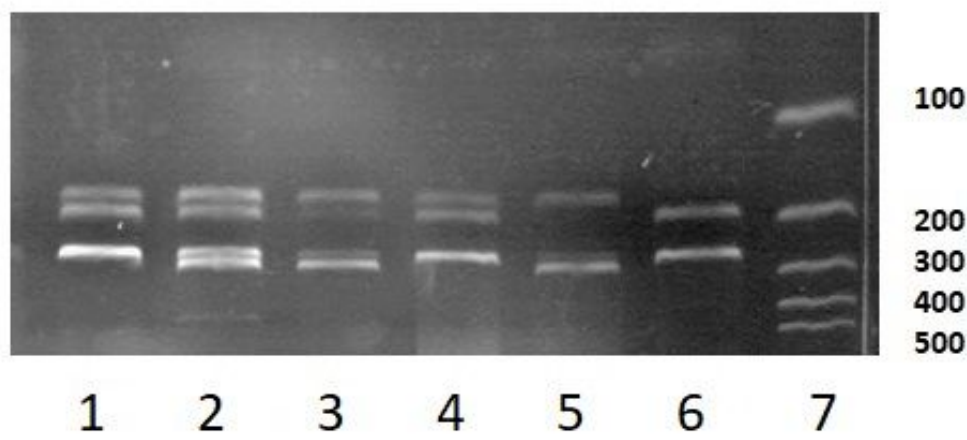

\section{Figure 1}

Representation of restriction digestion with Taql. Lane 1, 2, 4: heterozygous with $23+171+194+268$. Lane 3 and 5 : homozygous mutant with $23+171+268$. Lane 6: homozygous wild with $194+268$. Lane $7: 10$ to 500 bp DNA marker.

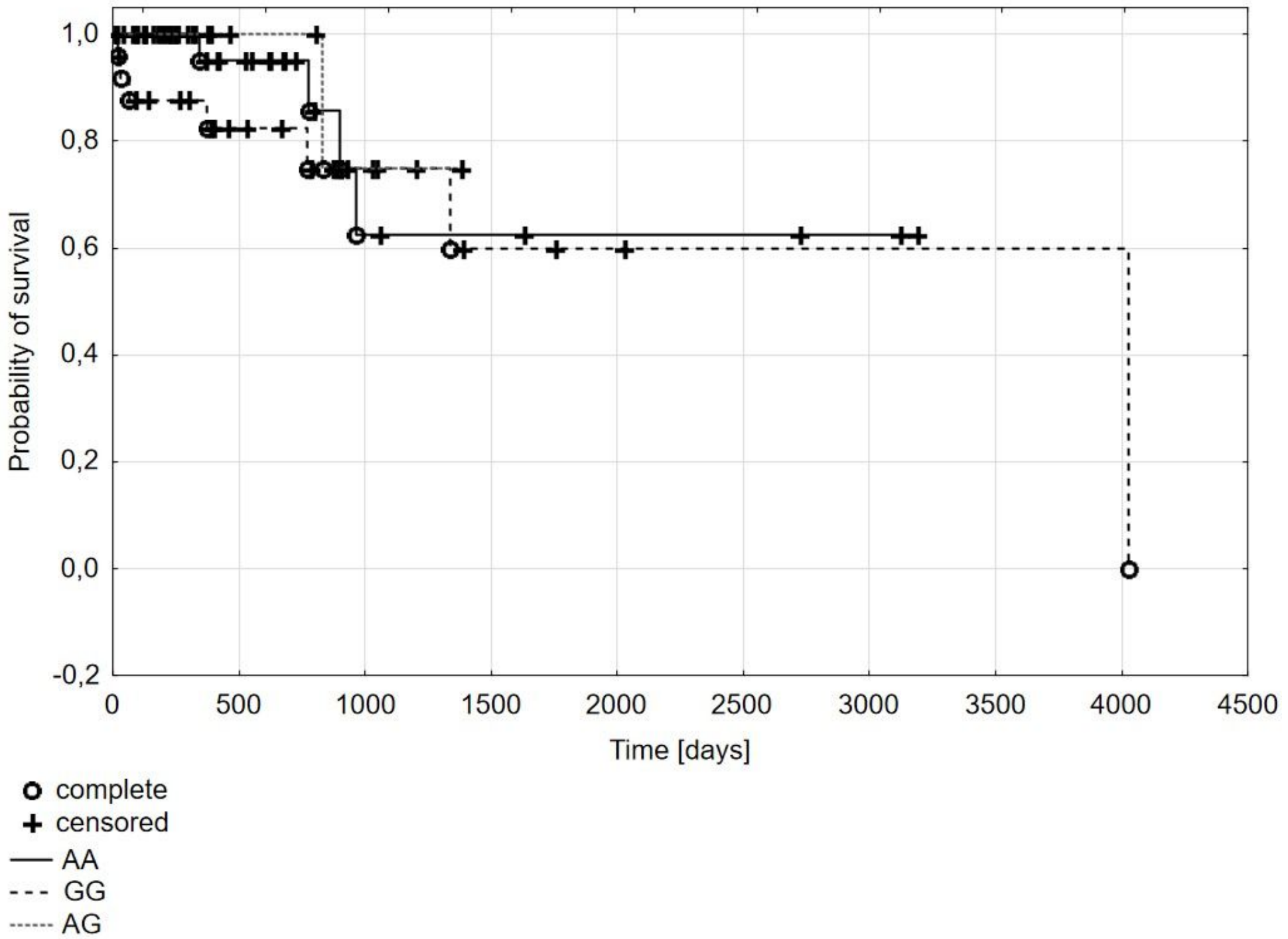

Figure 2

Kaplan-Meier plot for multiple myeloma patients with different genotypes for A388G polymorphism of the SLC01B1 gene. 


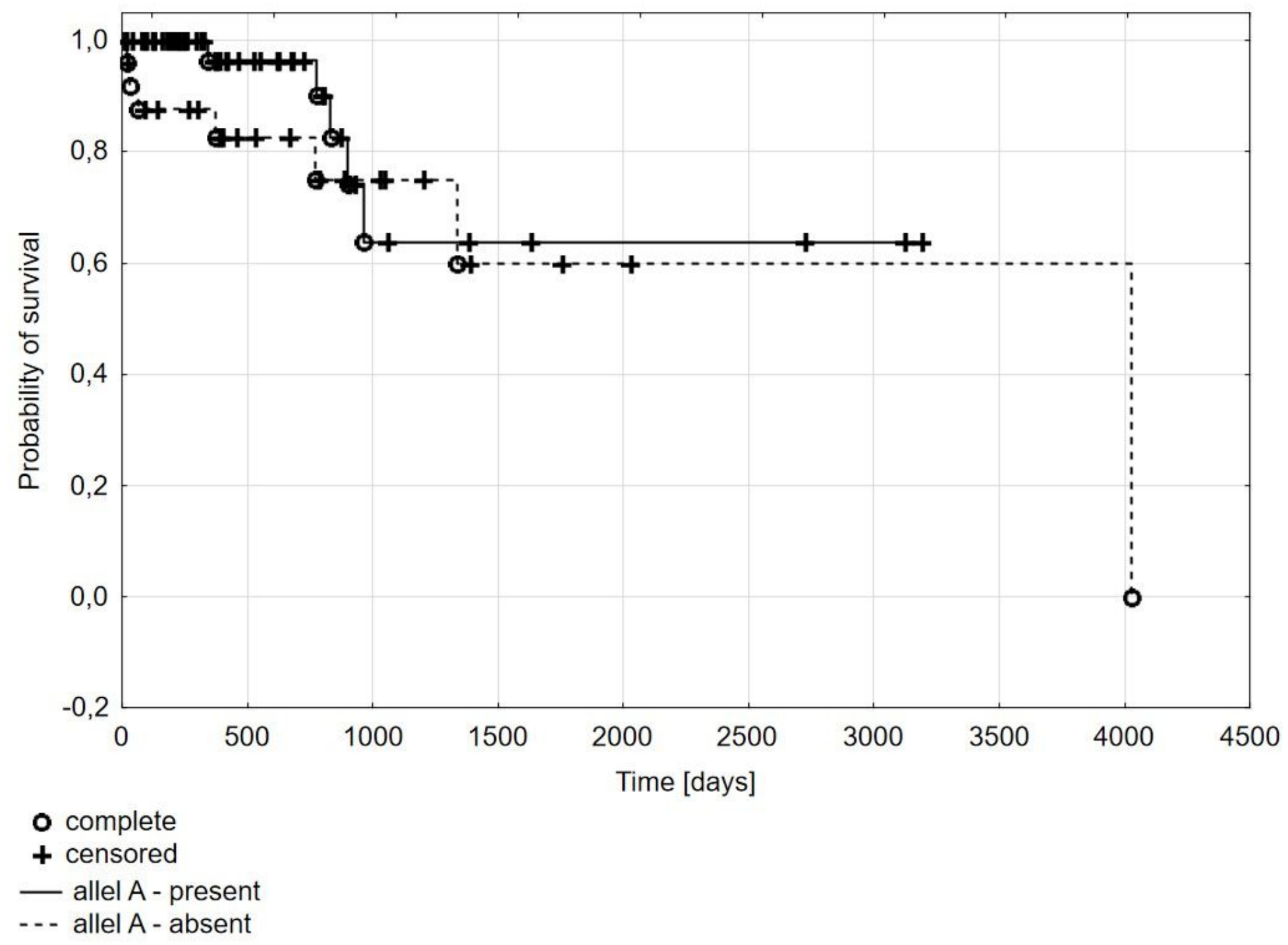

Figure 3

Kaplan-Meier plot for multiple myeloma patients with the present/ absent A allele in the A388G polymorphism of the SLC01B1 gene. 


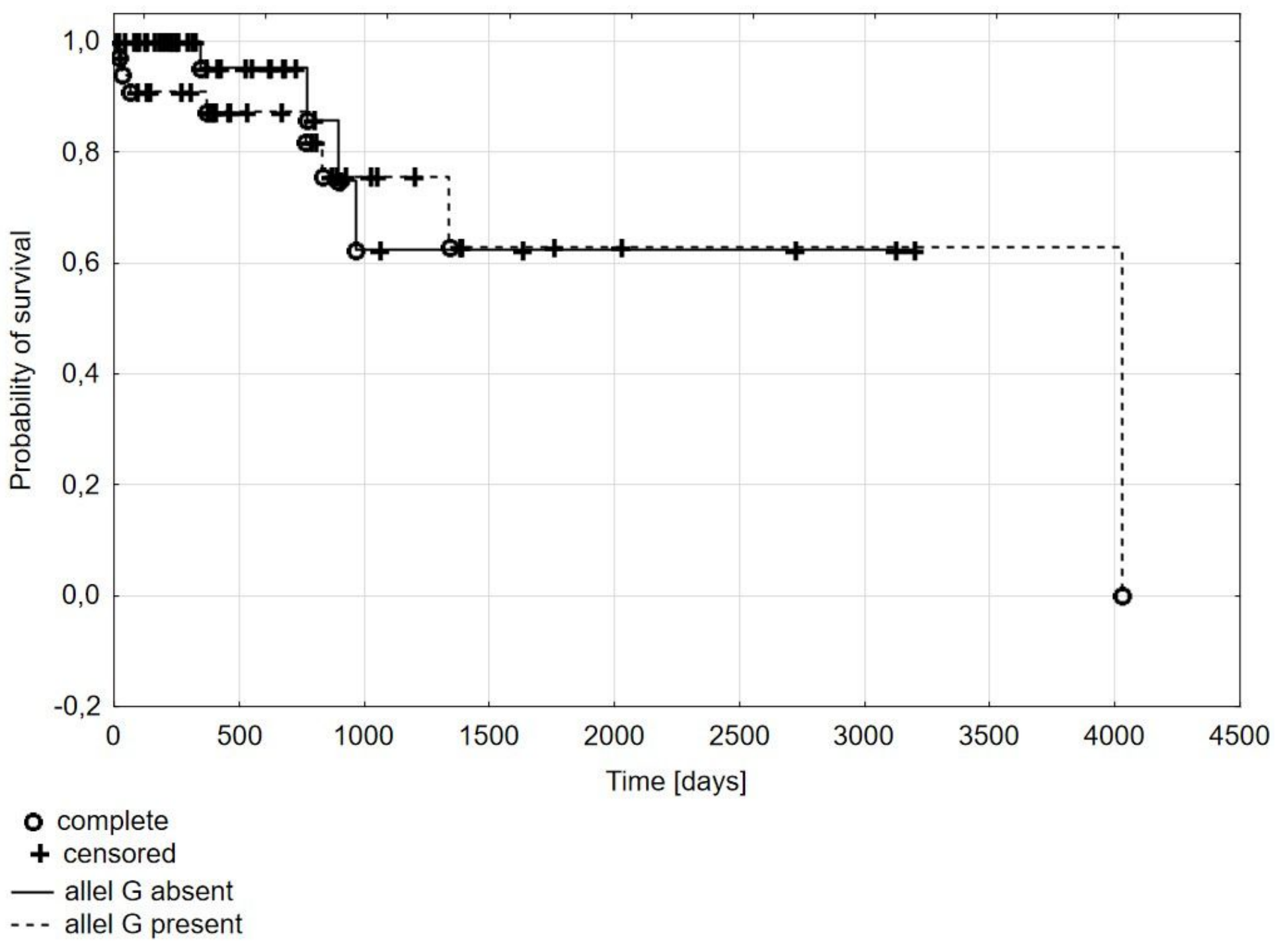

Figure 4

Kaplan-Meier plot for multiple myeloma patients with the present/ absent $\mathrm{G}$ allele in the A388G polymorphism of the SLCO1B1 gene. 